\title{
Cohesin dynamic association to chromatin and interfacing with replication forks in genome integrity maintenance.
}

\author{
Sara Villa-Hernández ${ }^{1,2}$ \& Rodrigo Bermejo ${ }^{1 *}$. \\ ${ }^{1}$ Centro de Investigaciones Biológicas (CIB-CSIC), Calle Ramiro de Maeztu 9, 28040, Madrid, Spain. \\ ${ }^{2}$ Current address: Wolfson Centre for Age-Related Diseases, King's College London, London SE1 1UL, \\ UK \\ *Correspondence to: rodrigo.bermejo@csic.es
}

\begin{abstract}
Proliferating cells need to accurately duplicate and pass their genetic material on to daughter cells. Problems during replication and partition challenge the structural and numerical integrity of chromosomes. Diverse mechanisms, as the DNA replication checkpoint, survey the correct progression of replication and couple it with other cell cycle events in order to preserve genome integrity. The structural maintenance of chromosomes (SMC) cohesin complex primarily contributes to chromosome duplication by mediating the tethering of newly replicated sister chromatids, thus assisting their equal segregation in mitosis. In addition, cohesin exerts important functions in genome organization, gene expression and DNA repair. These are determined by cohesin's ability to bring together different DNA segments and, hence, by the fashion and dynamics of its interaction with chromatin. It recently emerged that cohesin contributes to the protection of stalled replication forks through a mechanism requiring its timely mobilization from unreplicated DNA and relocation to nascent strands. This mechanism relies on DNA replication checkpoint-dependent cohesin ubiquitylation and promotes nascent sister chromatid entrapment, likely contributing to preserve stalled replisome-fork architectural integrity. Here we review how cohesin dynamic association to chromatin is controlled through post-translational modifications to dictate its functions during chromosome duplication. We also discuss recent insights on the mechanism that mediates interfacing of replisome components with chromatin-bound cohesin and its contribution to the establishment of sister chromatid cohesion and the protection of stalled replication forks.
\end{abstract}

\section{Key words}

Cohesin, DNA replication, Sister Chromatid Cohesion, replication forks, DNA replication checkpoint, cell cycle, genome integrity 


\section{Introduction}

In eukaryotes most of genetic information is contained in chromosomal DNA that must be accurately duplicated and partitioned every cell cycle. Chromosome replication initiates at multiple discrete sites, known as replication origins, in which two replication forks emerge to progress away until termination occurs, generally by convergence with forks coming from neighbouring origins (Siddiqui et al. 2013). Replication requires the unwinding of chromatin to provide a single stranded DNA (ssDNA) template for replicative polymerases Pol $\varepsilon$ and Pol $\delta$, primarily dedicated to leading and lagging strands synthesis, respectively (Doublié and Zahn 2014; Lujan et al. 2016). DNA unwinding is performed by the CMG (Cdc45-MCM-GINS) helicase complex which associates a number of additional factors to conform the replisome. Besides providing ssDNA templates, this complex molecular machine is in charge of the synthesis of leading and lagging strands, the coupling of DNA unwinding with DNA synthesis (through factors such as Ctf4/AND-1) and the maturation of discontinuous lagging strands (for instance by action of the FEN1 and Dna2 nucleases) (Villa et al. 2016; Kurat et al. 2017; Yeeles et al. 2017). Replication fork progression re-shapes chromosome structure, as helicase action requires the eviction of nucleosomes (subsequently re-assembled on nascent DNA duplexes) and imposes changes in the topology and higher order organization of DNA molecules (Bell and Labib 2016). Different factors associate to replisomes to mediate nucleosome removal and re-assembly during replication (such as the FACT complex or the Asf1 histone chaperon) and relax replication-driven torsional stress (i.e. DNA topoisomerases I and II) (Bermejo et al. 2007; Alabert et al. 2014). In addition, replisome progression is intimately intertwined with the establishment of sister chromatid cohesion (SCC), which is maintained by the Structural Maintenance of Chromosomes (SMC) cohesin complex (Carretero et al. 2010). Newly synthesized chromatids are embraced and locked by cohesin molecules in the wake of replication forks, in a scarcely known process that requires the function of several replisome factors, ensuring their close spatial association until anaphase (Mehta et al. 2012). Sister chromatid cohesion is itself important for the correct execution of mitosis, as it contributes to establishing bipolar kinetochore attachment and prevents premature separation of sister chromatids by pulling forces of spindle microtubules (Hirano 2015).

Conditions that impede DNA unwinding or synthesis, collectively known as replication stress, can stall the progression of replication forks and are thought to determine structural changes in the replisome-fork dynamic architecture (Branzei and Foiani 2010; Jossen and Bermejo 2013). Such 
changes are monitored by DNA replication checkpoint kinases, Mec1/ATR and Rad53/CHK1, which trigger a cellular response aimed at protecting genome integrity, prominently by preserving the proficiency for DNA synthesis of stalled forks (De Piccoli et al. 2012). Prolonged fork stalling, particularly in the absence of a functional checkpoint response, can lead to the formation of DNA breaks owing to fork collapse and/or nucleolytic processing of replication intermediates (Colosio et al. 2016; Pasero and Vindigni 2017). In addition, attempts to segregate incompletely replicated chromosomes can lead to missegregation and generate DNA breaks during mitosis (Fujita et al. 2013; Fragkos and Naim 2017). Replication stress-related DNA breaks have been proposed to promote tumorigenesis by driving the transformation of premalignant cells (Lecona and Fernándezcapetillo 2014; Gaillard et al. 2015).

Cohesin has been found to be enriched at replication forks both under normal and replication stress conditions (Tittel-Elmer et al. 2012; Ribeyre et al. 2016). This association likely relates to the recently discovered requirement for cohesin function to promote efficient fork progression upon stalling by the presence of damaged DNA templates, dNTPs depletion or at hard-to-replicate telomeric regions (Remeseiro et al. 2012; Tittel-Elmer et al. 2012). Cohesin also aids coping with DNA damage during S and G2 phases of the cell cycle, in which a close association of sister chromatids is thought to facilitate error-free bypass of lesions blocking DNA polymerases and repair of DNA breaks by homologous recombination through the copying of the identical genetic information on the sister chromatid (Ström et al. 2004; McAleenan et al. 2013). Hence, cohesin plays key roles in preventing genomic instability during chromosome duplication, both promoting DNA integrity during replication and a balanced chromosome segregation in mitosis. Here we review how the dynamic association of cohesin with chromatin is controlled to direct its functions in the chromosome duplication cycle. We also discuss recent insight on how interfacing between chromatin-bound cohesin and replication forks is achieved, as well as its contribution to genome integrity maintenance in conditions of replication stress.

\section{Dynamics of cohesin association to chromatin during the chromosome duplication cycle.}

The core cohesin complex is constituted by two SMC subunits, Smc1/SMC1 and Smc3/SMC3, which associate through their hinge domains and extrude long coiled-coil domains to form a "V-like" structure (Figure 1). An Scc1/RAD21 alpha-kleisin subunit interacts with Smc1 and Smc3 ATPase heads, at the end of coiled-coil domains, conforming a closed ring-like structure that can 
topologically embrace DNA molecules (Haering et al. 2002; Gligoris and Löwe 2016). In addition, accessory subunits Scc3 (ortholog of SA1 and SA2 in human) and Pds5 (PDS5A and PDS5B in human) interact with the core cohesin complex through Scc1 and influence the association of cohesin to chromatin (Michaelis et al. 1997; Sutani et al. 2009; Chan et al. 2013). The precise way in which the ring-shaped cohesin complexes interact with and lock in DNA molecules is not yet understood. Several models have been proposed that vary on whether one or more cohesin complexes associate different DNA segments and in the way cohesin complexes are arranged while embracing DNA (Kurze et al. 2011; Skibbens 2016). The embraced segments can belong to the same DNA molecule (as in the case of chromatin loop extrusion or the association of promoter and enhancer elements) or to different ones (to mediate sister chromatid cohesion, to facilitate DNA repair or to promote homologous chromosome pairing in meiosis) (Ström et al. 2004; Kagey et al. 2010; Dorsett 2011; Ding et al. 2016; Haarhuis et al. 2017). It is generally assumed that cohesin functions rely on the complex's ability to topologically associate DNA segments in a stable manner. For this reason, the control of the dynamics and topology of the interaction of cohesin with chromatin is thought to be crucial for the regulation of cohesin-mediated processes.

Association of cohesin to chromosomal DNA is indeed dynamic during the cell cycle and is influenced by post-translational modifications and the action of accessory factors (Figure 2). The cohesin ring is recruited to DNA before S-phase, in G1 in budding yeast and after telophase in mammalian cells, by action of the Scc2/Scc4 complex (NIPBL/MAU2 in human). Cohesin loading implies the entrance of DNA molecules into the cohesin structure, where the Smc3-Smc1 hinge and Smc3-Scc1 interfaces have been proposed to permit entry into the complex (Murayama and Uhlmann 2015). The precise mechanism by which cohesin is loaded onto DNA is not yet fully understood, but recent insight into Scc2 structure supports the notion that multiple contacts between Scc2 and cohesin are essential for this process (Chao et al. 2017). Scc2 was proposed to stabilize two sensory lysines (K112 and K113 in budding yeast) close to the ATPase head domain of Smc3, promoting ATP hydrolysis and the opening of an entry gate for DNA (Murayama and UhImann 2015). This association of cohesin to chromatin is labile and can be reversed due to the action of regulators such as Wpl1/WAPL and Pds5, which are thought to dislodge the Smc3-Scc1 interface to permit DNA exit from the cohesin structure (Kueng et al. 2006; Sutani et al. 2009; Chan et al. 2012). In S phase a fraction of cohesin becomes stably associated to chromatin, thought to correspond to molecules embracing newly replicated sister chromatids, by action of the fork-associated Eco1 acetyltransferase (ESCO1 and ESCO2 in human) that acetylates K112 and K113 on Smc3 (Nasmyth 2011; Lopez-Serra et al. 2013). These modifications likely render the Smc3-Scc1 interface refractory to Wpl1/Pds5 action, precluding DNA exit from the complex, and determine a fork-coupled establishment of sister chromatid cohesion. 
Tethering of sister chromatids is maintained until mitosis in which cleavage of the kleisin subunit triggers chromosome segregation (Morales and Losada 2018). In anaphase, the APC/Cdh1 E3 ubiquitin ligase promotes the degradation of securin, an inhibitor of the separase protease responsible of cleaving Scc1/RAD21. Separase activation mediates the removal of centromereassociated cohesin complexes, an event permitting the separation of sister chromatids (UhImann et al. 1999; Alexandru et al. 2001). While this is the main mechanism for cohesion dissolution in yeast cells, additional mechanisms have been described in higher eukaryotes. In vertebrate cells, cohesin is removed from chromosome arms during prophase, in a mechanism mediated by the Plk1 kinase. Phosphorylation of the SA subunit by Plk1 is required for cohesin dissociation, in a process also requiring WAPL1 function (Hauf et al. 2005). It has been proposed that cohesin removal from chromosome arms may facilitate sister chromatid resolution through decatenation by DNA topoisomerase II (Losada et al. 2002), which has been proposed to also crosstalk with Smc3 acetylation (Lin and O'Connell 2017). This prophase pathway would be restricted from dissolving centromeric cohesion by action of the cohesin protector Shugoshin 1 (SGO1) and the protein phosphatase 2A (PP2A) that counteract cohesin phosphorylation mainly at centromeres (Liu et al. 2013). It has also been recently suggested that, in yeast cells, Smc3 deacetylation by Hos1 in early anaphase is required for the opening of a DNA exit gate and cohesin removal (Li et al. 2017). In addition, a function in recycling Smc3 for its use in the following cell cycle has been attributed to the Hos1 deacetylase (Beckouet et al. 2010; Xiong et al. 2010). Post-translational modifications play also important roles in modulating cohesin association to chromatin in response to DNA breaks. Cohesin complexes are recruited to chromatin surrounding double stranded DNA breaks, presumably to maintain the proximity between broken DNA ends and thus facilitate repair through homologous recombination (Gelot et al. 2016). DNA damage-induced cohesion requires Eco1 acetylase function, although in this context Scc1, instead of Smc3, seems the key target (Heidinger-Pauli et al. 2009). Scc1 acetylation requires previous checkpoint-dependent phosphorylation, indicating that in this context cohesin regulation by the DNA damage response is primarily exerted through its kleisin subunit. A fascinating picture is therefore emerging on the interplaying roles of post-translational modifications in modulating cohesin functional association to chromatin along the cell cycle, that may operate differently at specific genomic regions or following DNA damage.

\section{Interfacing of chromatin-associated cohesin with replisome factors upon engagement by replication forks}


Since cohesin is loaded onto chromatin prior to replication initiation, advancing forks are bound to encounter cohesin molecules on template DNA. While interfacing of replication forks with cohesin complexes likely impacts on cohesion establishment and fork integrity, the mechanism through which it is achieved is only beginning to be understood. Three different fates have been proposed for cohesin during replication (UhImann 2016). One possibility is that replication machineries slide through the cohesin ring, so that sister chromatids are "synthesized into" cohesin achieving their passive entrapment. A second possibility is that an opening in the ring structure is induced when replication forks engage cohesin leading to a transient dislodging of cohesin from chromatin, which may however remain in proximity of the replisome, and its subsequent reloading around sister chromatids. Lastly, cohesin may be removed from chromatin upon engagement by replication forks and unrelated cohesin molecules loaded around nascent sister chromatids.

Recent studies argue for chromatin-bound cohesin being transiently mobilized in front of replication forks and transferred to nascent duplexes. In human cells lacking WAPL, cohesin molecules remain associated to chromosomes throughout S-phase, indicating that nascent sister chromatid entrapment can be achieved on the expense of "recycled" cohesin, already associated to chromatin prior to replication (Rhodes et al. 2017). Work on budding yeast also provided insight on how cohesin translocation behind forks might occur at the molecular level (Frattini et al. 2017). In Sphase cells, ubiquitylated forms of the Smc1, Smc3 and Scc1 cohesin subunits were detected in a manner dependent on the Rsp5E3 ubiquitin ligase and its arrestin-like adaptor Bul2. Mutants having impaired function of $\mathrm{Rsp}^{\mathrm{Bul} 2}$ or the $\mathrm{VCP} / \mathrm{Cdc} 48 / \mathrm{p} 97$ ubiquitin selective segregase show increased levels of cohesin association to chromatin nearby sites of active replication, suggestive of a defect in mobilizing cohesin from replicating chromatin. In contrast, when the association of fork proximal cohesin to BrdU-labelled newly synthesized DNA was assayed, it was found to be dramatically reduced in Rsp5 ${ }^{\text {Bul2 }}$ and Cdc48 mutants (Frattini et al. 2017). These observations suggest that ubiquitylation favours cohesin mobilization from unreplicated chromatin, which is required for its subsequent re-location to newly synthesized sister duplexes (Figure 2).

The molecular steps leading to cohesin mobilization from replicating chromatin remain obscure. Cdc48 targets ubiquitylated proteins and induces conformational changes that dislodge them from their cellular environment (Stolz et al. 2011; Dantuma and Hoppe 2012). Cdc48 has been involved in the extraction of ubiquitylated proteins from chromatin, including the CMG replicative helicase, the KU repair factor or RNA polymerase II (Harreman et al. 2009; Verma et al. 2011; Maric et al. 2014; van den Boom et al. 2016). Hence, it is reasonable to think that, also in the case of cohesin, ubiquitylation may trigger or be a prerequisite for extraction from chromatin when the complex is engaged by replication forks. In this view, Cdc48 would target ubiquitylated cohesin and reshape its 
structure to favour the opening of a DNA exit gate and the release of the complex from DNA. Of note, a role for $\mathrm{Cdc} 48$ in mobilizing the $\mathrm{SMC}$ condensin complex to promote its dynamic interaction with chromatin was recently described (Robellet et al. 2017; Thattikota et al. 2018). Mutations in $\mathrm{Rsp}^{\mathrm{Bul} 2}$ and $\mathrm{Cdc} 48$ impairing cohesin mobilization confer sensitivity to replication stress inducing agents, which is genetically linked to cohesin function in replication fork protection (Frattini et al. 2017). Wpl1 ablation also confers HU sensitivity, which is epistatic to that of mutations impairing cohesin ubiquitylation and mobilization. It is tantalizing to speculate, based on these genetic interactions, that conformational changes induced by Cdc48 favour Wpl1-mediated opening of a gate at the Smc3-Scc1 interface to mediate cohesin release from template chromatin. Identification of residues ubiquitylated in an $\mathrm{Rsp}^{\mathrm{Bu} \mathrm{I}^{2}}$-dependent manner on the different cohesin subunits may provide insight on the structural remodelling of the complex and putative opening of DNA exit gates.

A key aspect of the transient opening model is how would mobilized cohesin be shuttled for reloading. This may occur through transient physical interaction of evicted cohesin with replisome components, which may also contribute to integrate cohesin translocation with the synthesis of nascent strands. In budding yeast, replisome factors as Ctf4/AND-1, thought to act as a hub coupling the GINS component of the CMG helicase with lagging strand polymerases, and Ctf18 or Elg1/ATAD5, components of alternative Replication Factor $C$ complexes mediating the loading/unloading of the PCNA (Proliferating Cell Nuclear Antigen) replicative polymerase processivity factor, are necessary for efficient cohesion establishment (Maradeo and Skibbens 2010; Lai et al. 2012; Samora et al. 2016). This evidence supports the notion that cohesin translocation to nascent chromatids may be influenced by replisome dynamic architectural features, specifically related to the coordination between DNA unwinding and processive nascent strand synthesis (Rudra and Skibbens 2012). Intriguingly, Ctf4 is also involved in attracting the Chl1 (homolog of human ChIR1/DDX11 and BACH1/BRIP1/FANCJ) helicase to the replisome, which is enigmatically required for SCC establishment in a fashion independent of its catalytic activity (Samora et al. 2016). Also intriguing is the question how would cohesin be loaded back to entrap newly synthesized chromatids. In vitro evidence using purified fission yeast cohesin, indicates that capture of a second DNA by cohesin molecules that already embrace a DNA duplex is more efficient when using ssDNA as a substrate (Murayama et al. 2018). These observations may have important implications for cohesin interfacing with replication forks, as mobilized cohesin molecules might first embrace double stranded DNA, promptly formed by CMG-coupled leading strand synthesis, and subsequently capture ssDNA on the lagging strand template prior to its replication. In this view, coordination of cohesin loading and leading/lagging strand synthesis machineries might play an important role for the establishment of sister chromatid cohesion. In vitro, second DNA capture depends on the 
Mis4/Ssl3 (Scc2/Scc4) complex, so far the only know factor able to recruit cohesin to chromatin. It is therefore reasonable to think that this complex may associate to replication sites to mediate cohesin "recycling" and entrap nascent chromatid in two consecutive steps. However, it cannot be formally excluded that replisome-associated cohesin may be re-loaded through a different mechanism. In this respect, it has been reported that cohesin accumulation nearby stalled replication forks depends on the MRX (Mre11-Rad50-Xrs2) DNA repair complex (Tittel-Elmer et al. 2012). This complex is also characterized by the presence of long coiled-coil domains ending in ATPase heads in its Mre11 subunit and has been proposed to tether DNA break ends together (Seeber et al. 2016). It is however not unclear, whether this MRX-dependency may reflect a function in cohesin recruitment to sister chromatids or instead a requirement to prevent cohesin release, perhaps due to sliding through free DNA ends resulting from fork collapse in the absence of the complex.

\section{Contribution of the interfacing of cohesin with replisome components to the stabilization of stalled forks}

The active replisome is highly dynamic and situations inducing replication stress are thought to result in changes in replisome-fork structure (Sogo et al. 2002; Gan et al. 2017; Yeeles et al. 2017). For instance, inhibition of DNA synthesis leads to uncoupling of leading/lagging strand synthesis and DNA unwinding, resulting in the formation extended single strand DNA stretches at stalled forks (Ball et al. 2005; Byun et al. 2005). The DNA replication checkpoint kinase Mec1/ATR directly monitors these changes and elicits a cellular response aimed at protecting genome integrity (Zou and Elledge 2003). Amongst checkpoint-mediated effects, protection of fork integrity is retained as the key function influencing cell viability in response to replication stress (Tercero and Diffley 2001), proposed to be exerted through direct regulation of replisome components function as opposed to prevention of replisome breakdown (De Piccoli et al. 2012). The precise mechanisms through which checkpoint kinases preserve the proficiency for DNA synthesis of stalled fork-replisome ensemble are not still fully understood, though they likely imply the suppression of nascent strands displacement from DNA polymerases (e.g. during fork reversal) and/or nucleolytic processing (Cottaramusino et al. 2005; Bermejo et al. 2011; Jossen and Bermejo 2013; Colosio et al. 2016). Additional replisome-associated factors contribute to promote the functionality of replication forks by either directly removing obstacles to fork progression (as Rrm3, Pif1/PIF1 and Sen1/SENATAXIN helicases) or by somehow providing structural stability to paused replisomes (as the Mrc1/CLASPINTof1/TIMELESS-Csm3/TIPIN replisome protection complex components) (Katou et al. 2003; Calzada et al. 2005; Tourrière et al. 2005; Azvolinsky et al. 2006; Bando et al. 2009; Alzu et al. 2012). 
Strikingly, mutations in checkpoint and replisome stabilizing factors also result in defects in sister chromatid cohesion establishment (Xu et al. 2007), further supporting the notion that altered replisome dynamics impact on nascent chromatid entrapment and locking. Similarly, several factors contributing to the dynamic organization of the replisome (e.g. Ctf4, Ctf18 and Elg1), also required for SCC establishment, are important for the stable progression of stressed replication forks (Bellaoui et al. 2003; Poli et al. 2012; Villa et al. 2016). Thus, stable replisome-fork architecture appears to be a critical requirement for both preserving stalled replication forks capacity to resume DNA synthesis and for effective cohesion establishment.

Mutations impairing cohesin function, mobilization from chromatin or translocation to and locking of newly synthesized sister chromatids confer sensitivity to agents inducing replication stress, stalled fork progression defects and impaired sister chromatid cohesion (Frattini et al. 2017). Hence, replication defects upon impaired cohesin function are likely to also stem from inability to stabilize stalled fork architecture. Of note, impairment of Eco1 activity confers HU sensitivity in a fashion epistatic to that of $\mathrm{Rsp}^{\mathrm{Bul} 2}$ and $\mathrm{Cdc} 48$ mutations, indicating that cohesin acetylation at stalled replication forks, and presumably a close association of sister chromatids, is important for this mechanism. Hence, a likely role for cohesin may be to provide "constraining support" to prevent the disarray of replisome factors in association with stalled nascent chromatids (Tittel-Elmer et al. 2009), either passive owing to cessation of processive DNA synthesis or induced by topological transitions affecting replication forks (Bermejo et al. 2011). Collectively, this evidence suggests a scenario in which factors supporting the dynamic replisome-fork architecture and promoting cohesin mobilization interplay to achieve a productive molecular interfacing resulting in the efficient entrapment of nascent chromatids. Interestingly, both the interaction of Rsp5 $5^{\text {Bul2 }}$ with cohesin and the detection of ubiquitylated cohesin forms depend on Mec1 signalling (Frattini et al. 2017) and checkpoint-dependent phosphorylation of cohesin has been observed in different organisms (Yazdi et al. 2002; Heidinger-Pauli et al. 2009; Bauerschmidt et al. 2011), suggesting that fork-cohesin interfacing and cohesin-mediated fork protection might be directly promoted by the checkpoint response.

\section{Concluding remarks}

Cohesin plays key roles in chromosome organization and in the maintenance of genome integrity. Mutations in genes coding for cohesin subunits or its regulatory factors cause human disease and have been associated to different types of cancer (Solomon et al. 2014; Zakari et al. 2015; Hill et al. 2016; Koninck and Losada 2016). How cohesin dysregulation contributes to cancer development is 
however not yet clear. As argued, impaired cohesin function can negatively impact on genome instability in at least three direct ways: by promoting aneuploidy due to unbalanced chromosome segregation, by favouring the formation of stalled fork-driven DNA breaks and by increasing the genotoxic impact of DNA lesions due to faulty repair. Importantly, both replication stress-related DNA damage and aneuploidy are regarded as drivers of the malignant transformation process (Bartkova et al. 2005; Bartek and Lukas 2007; Wenzel and Singh 2018). The work here discussed provides a framework to understand how dynamic interfacing of cohesin with replication forks may influence both fork stability and the establishment of sister chromatid cohesion. It will be exciting in following years to advance our understanding on the molecular mechanisms mediating cohesin-fork interfacing and gain insight on whether this process is affected by cancer-related cohesin mutations.

\section{Acknowledgements}

We apologise for all the relevant findings and studies that could not be included in this review. This work was supported by the Ministry of Economy, Industry and Competitiveness - MINECO (BFU201452529-R and BFU2017-87013-R to R.B) and the Spanish Formación del Personal Investigador (FPI) program (to S.V-H.).

\section{References}

Alabert C, Bukowski-Wills J-C, Lee S-B, et al (2014) Nascent chromatin capture proteomics determines chromatin dynamics during DNA replication and identifies unknown fork components. Nat Cell Biol 16:281-293. doi: 10.1038/ncb2918

Alexandru G, Uhlmann F, Mechtler K, et al (2001) Phosphorylation of the cohesin subunit Scc1 by Polo/Cdc5 kinase regulates sister chromatid separation in yeast. Cell 105:459-72.

Alzu A, Bermejo R, Begnis M, et al (2012) Senataxin associates with replication forks to protect fork integrity across RNA-polymerase-II-transcribed genes. Cell 151:835-46. doi: 10.1016/j.cell.2012.09.041

Azvolinsky A, Dunaway S, Torres JZ, et al (2006) The S. cerevisiae Rrm3p DNA helicase moves with the replication forkand affects replication of allyeast chromosomes. Genes Dev 20:3104-3116. doi: 10.1101/gad.1478906

Ball HL, Myers JS, Cortez D (2005) ATRIP Binding to Replication Protein A-Single-stranded DNA Promotes ATR - ATRIP Localization but Is Dispensable for Chk1 Phosphorylation. 16:23722381. doi: $10.1091 / \mathrm{mbc}$.E04

Bando M, Katou Y, Komata M, et al (2009) Csm3, Tof1, and Mrc1 form a heterotrimeric mediator complex that associates with DNA replication forks. J Biol Chem 284:34355-34365. doi: 10.1074/jbc.M109.065730 
Bartek J, Lukas J (2007) DNA damage checkpoints: from initiation to recovery or adaptation. Curr Opin Cell Biol 19:238-45. doi: 10.1016/j.ceb.2007.02.009

Bartkova J, Horejsí Z, Koed K, et al (2005) DNA damage response as a candidate anti-cancer barrier in early human tumorigenesis. Nature 434:864-870. doi: 10.1038/nature03482

Bauerschmidt C, Woodcock M, Stevens DL, et al (2011) Cohesin phosphorylation and mobility of SMC1 at ionizing radiation-induced DNA double-strand breaks in human cells. Exp Cell Res 317:330-337. doi: 10.1016/j.yexcr.2010.10.021

Beckouet F, Hu B, Roig MB, et al (2010) An Smc3 Acetylation Cycle Is Essential for Establishment of Sister Chromatid Cohesion. Mol Cell 39:689-699. doi: 10.1016/j.molcel.2010.08.008

Bell SP, Labib K (2016) Chromosome Duplication in Saccharomyces cerevisiae. Genetics 203:10271067. doi: 10.1534/genetics.115.186452

Bellaoui M, Chang M, Ou J, et al (2003) Elg1 forms an alternative RFC complex important for DNA replication and genome integrity. EMBO J 22:4304-4313. doi: 10.1093/emboj/cdg406

Bermejo R, Capra T, Jossen R, et al (2011) The replication checkpoint protects fork stability by releasing transcribed genes from nuclear pores. Cell 146:233-246. doi: 10.1016/j.cell.2011.06.033

Bermejo R, Doksani Y, Capra T, et al (2007) Top1- and Top2-mediated topological transitions at replication forks ensure fork progression and stability and prevent DNA damage checkpoint activation. Genes Dev 21:1921-1936. doi: 10.1101/gad.432107

Branzei D, Foiani M (2010) Maintaining genome stability at the replication fork. Nat Rev Mol Cell Biol 11:208-19. doi: 10.1038/nrm2852

Byun TS, Pacek M, Yee MC, et al (2005) Functional uncoupling of MCM helicase and DNA polymerase activities activates the ATR-dependent checkpoint. Genes Dev 19:1040-1052. doi: 10.1101/gad.1301205

Calzada A, Hodgson B, Kanemaki M, et al (2005) Molecular anatomy and regulation of a stable replisome eukaryotic DNA at a paused replication fork. Genes Dev 19:1905-1919. doi: 10.1101/gad.337205

Carretero M, Remeseiro S, Losada A (2010) Cohesin ties up the genome. Curr Opin Cell Biol 22:7817. doi: $10.1016 /$ j.ceb.2010.07.004

Chan K-L, Gligoris T, Upcher W, et al (2013) Pds5 promotes and protects cohesin acetylation. Proc Natl Acad Sci 110:13020-13025. doi: 10.1073/pnas.1306900110

Chan K-L, Roig MB, Hu B, et al (2012) Cohesin's DNA exit gate is distinct from its entrance gate and is regulated by acetylation. Cell 150:961-74. doi: 10.1016/j.cell.2012.07.028

Chao WCH, Murayama Y, Muñoz S, et al (2017) Structure of the cohesin loader Scc2. Nat Commun 8:13952. doi: $10.1038 /$ ncomms 13952

Colosio A, Frattini C, Pellicanò G, et al (2016) Nucleolytic processing of aberrant replication intermediates by an Exo1-Dna2-Sae2 axis counteracts fork collapse-driven chromosome instability. Nucleic Acids Res 12:gkw858. doi: 10.1093/nar/gkw858

Cotta-ramusino C, Fachinetti D, Lucca C, et al (2005) Exo1 processes stalled replication forks and counteracts fork reversal in checkpoint-defective cells. Mol Cell 17:153-9. doi: 10.1016/j.molcel.2004.11.032 
Dantuma NP, Hoppe T (2012) Growing sphere of influence: Cdc48/p97 orchestrates ubiquitindependent extraction from chromatin. Trends Cell Biol 22:483-91. doi:

10.1016/j.tcb.2012.06.003

De Piccoli G, Katou Y, Itoh T, et al (2012) Replisome Stability at Defective DNA Replication Forks Is Independent of S Phase Checkpoint Kinases. Mol Cell 45:696-704. doi:

10.1016/j.molcel.2012.01.007

Ding DQ, Haraguchi T, Hiraoka Y (2016) A cohesin-based structural platform supporting homologous chromosome pairing in meiosis. Curr Genet 62:499-502. doi: 10.1007/s00294-016-0570-x

Dorsett D (2011) Cohesin: genomic insights into controlling gene transcription and development. Curr Opin Genet Dev 21:199-206. doi: 10.1016/j.gde.2011.01.018

Doublié S, Zahn KE (2014) Structural insights into eukaryotic DNA replication. Front Microbiol 5:444. doi: $10.3389 /$ fmicb.2014.00444

Fragkos M, Naim V (2017) Rescue from replication stress during mitosis. Cell Cycle 16:613-633. doi: 10.1080/15384101.2017.1288322

Frattini C, Villa-Hernández S, Pellicanò G, et al (2017) Cohesin Ubiquitylation and Mobilization Facilitate Stalled Replication Fork Dynamics. Mol Cell 68:758-772.e4. doi:

10.1016/j.molcel.2017.10.012

Fujita M, Sasanuma H, Yamamoto KN, et al (2013) Interference in DNA Replication Can Cause Mitotic Chromosomal Breakage Unassociated with Double-Strand Breaks. PLoS One. doi: 10.1371/journal.pone.0060043

Gaillard H, García-Muse T, Aguilera A (2015) Replication stress and cancer. Nat Rev Cancer 15:276289. doi: $10.1038 / \mathrm{nrc3916}$

Gan H, Yu C, Devbhandari S, et al (2017) Checkpoint Kinase Rad53 Couples Leading- and LaggingStrand DNA Synthesis under Replication Stress. Mol Cell 68:446-455.e3. doi: 10.1016/j.molcel.2017.09.018

Gelot C, Guirouilh-Barbat J, Le Guen T, et al (2016) The Cohesin Complex Prevents the End Joining of Distant DNA Double-Strand Ends. Mol Cell 15-26. doi: 10.1016/j.molcel.2015.11.002

Gligoris T, Löwe J (2016) Structural Insights into Ring Formation of Cohesin and Related Smc Complexes. Trends Cell Biol 26:680-693. doi: 10.1016/j.tcb.2016.04.002

Haarhuis JHI, van der Weide RH, Blomen VA, et al (2017) The Cohesin Release Factor WAPL Restricts Chromatin Loop Extension. Cell 169:693-707.e14. doi: 10.1016/j.cell.2017.04.013

Haering CH, Lo J, Hochwagen A, Nasmyth K (2002) Molecular Architecture of SMC Proteins and the Yeast Cohesin Complex. Mol Cell 9:773-788.

Harreman M, Taschner M, Sigurdsson S, et al (2009) Distinct ubiquitin ligases act sequentially for RNA polymerase II polyubiquitylation. Proc Natl Acad Sci U S A 106:20705-10. doi: 10.1073/pnas.0907052106

Hauf S, Roitinger E, Koch B, et al (2005) Dissociation of cohesin from chromosome arms and loss of arm cohesion during early mitosis depends on phosphorylation of SA2. PLoS Biol 3:0419-0432. doi: 10.1371/journal.pbio.0030069

Heidinger-Pauli JM, Unal E, Koshland D (2009) Distinct targets of the Eco1 acetyltransferase modulate cohesion in S phase and in response to DNA damage. Mol Cell 34:311-21. doi: 10.1016/j.molcel.2009.04.008 
Hill VK, Kim J-S, Waldman T (2016) Cohesin mutations in human cancer. Biochim Biophys Acta - Rev Cancer 1866:1-11. doi: 10.1016/j.bbcan.2016.05.002

Hirano T (2015) Chromosome Dynamics during Mitosis. Cold Spring Harb Perspect Biol 7:1-14. doi: 10.1101/cshperspect.a015792.

Jossen R, Bermejo R (2013) The DNA damage checkpoint response to replication stress: A Game of Forks. Front Genet 4:26. doi: 10.3389/fgene.2013.00026

Kagey MH, Newman JJ, Bilodeau S, et al (2010) Mediator and cohesin connect gene expression and chromatin architecture. Nature 467:430-5. doi: 10.1038/nature09380

Katou Y, Kanoh Y, Bando M, et al (2003) S-phase checkpoint proteins Tof1 and Mrc1 form a stable replication-pausing complex. Nature 424:1078-83. doi: 10.1038/nature01900

Koninck M De, Losada A (2016) Cohesin mutations in human cancer. Cold Spring Harb Perspect Med. doi: 10.1016/j.bbcan.2016.05.002

Kueng S, Hegemann B, Peters BH, et al (2006) Wapl Controls the Dynamic Association of Cohesin with Chromatin. Cell 127:955-967. doi: 10.1016/j.cell.2006.09.040

Kurat CF, Yeeles JTP, Patel H, et al (2017) Chromatin Controls DNA Replication Origin Selection, Lagging-Strand Synthesis, and Replication Fork Rates. Mol Cell 65:117-130. doi: 10.1016/j.molcel.2016.11.016

Kurze A, Michie K a, Dixon SE, et al (2011) A positively charged channel within the Smc1/Smc3 hinge required for sister chromatid cohesion. EMBO J 30:364-78. doi: 10.1038/emboj.2010.315

Lai MS, Seki M, Tada S, Enomoto T (2012) Rmi1 functions in S phase-mediated cohesion establishment via a pathway involving the Ctf18-RFC complex and Mrc1. Biochem Biophys Res Commun 427:682-6. doi: 10.1016/j.bbrc.2012.09.124

Lecona E, Fernández-capetillo O (2014) Replication stress and cancer : It takes two to tango. 329:2634. doi: 10.1016/j.yexcr.2014.09.019

Li S, Yue Z, Tanaka TU (2017) Smc3 Deacetylation by Hos1 Facilitates Efficient Dissolution of Sister Chromatid Cohesion during Early Anaphase. Mol Cell 68:605-614.e4. doi: 10.1016/j.molcel.2017.10.009

Lin SJ, O'Connell MJ (2017) DNA Topoisomerase II modulates acetyl-regulation of cohesin-mediated chromosome dynamics. Curr Genet 63:923-930. doi: 10.1007/s00294-017-0691-x

Liu H, Rankin S, Yu H (2013) Phosphorylation-enabled binding of SGO1-PP2A to cohesin protects sororin and centromeric cohesion during mitosis. Nat Cell Biol 15:40-49. doi: 10.1038/ncb2637

Lopez-Serra L, Lengronne A, Borges V, et al (2013) Budding yeast Wapl controls sister chromatid cohesion maintenance and chromosome condensation. Curr Biol 23:64-9. doi:

10.1016/j.cub.2012.11.030

Losada A, Hirano M, Hirano T (2002) Cohesin release is required for sister chromatid resolution, but not for condensin-mediated compaction, at the onset of mitosis. Genes Dev 16:3004-3016. doi: 10.1101/gad.249202

Lujan SA, Williams JS, Kunkel TA (2016) DNA Polymerases Divide the Labor of Genome Replication. Trends Cell Biol 26:640-654. doi: 10.1016/j.tcb.2016.04.012

Maradeo ME, Skibbens R V. (2010) Replication factor C complexes play unique pro- and antiestablishment roles in sister chromatid cohesion. PLoS One 5:1-9. doi: 
10.1371/journal.pone.0015381

Maric M, Maculins T, De Piccoli G, Labib K (2014) Cdc48 and a ubiquitin ligase drive disassembly of the CMG helicase at the end of DNA replication. Science (80- ) 346:1253596-1253596. doi: $10.1126 /$ science. 1253596

McAleenan A, Clemente-Blanco A, Cordon-Preciado V, et al (2013) Post-replicative repair involves separase-dependent removal of the kleisin subunit of cohesin. Nature 493:250-4. doi: $10.1038 /$ nature11630

Mehta GD, Rizvi SMA, Ghosh SK (2012) Cohesin: A guardian of genome integrity. Biochim Biophys Acta - Mol Cell Res 1823:1324-1342. doi: 10.1016/j.bbamcr.2012.05.027

Michaelis C, Ciosk R, Nasmyth K (1997) Cohesins: Chromosomal Proteins that Prevent Premature Separation of Sister Chromatids. Cell 91:35-45. doi: 10.1016/S0092-8674(01)80007-6

Morales C, Losada A (2018) Establishing and dissolving cohesion during the vertebrate cell cycle. Curr Opin Cell Biol in press:51-57. doi: 10.1016/j.ceb.2018.01.010

Murayama Y, Samora CP, Kurokawa Y, et al (2018) Establishment of DNA-DNA Interactions by the Cohesin Ring. Cell 1-13. doi: 10.1016/j.cell.2017.12.021

Murayama Y, Uhlmann F (2015) DNA Entry into and Exit out of the Cohesin Ring by an Interlocking Gate Mechanism. Cell 163:1628-1640. doi: 10.1016/j.cell.2015.11.030

Nasmyth K (2011) Cohesin: a catenase with separate entry and exit gates? Nat Cell Biol 13:1170-7. doi: $10.1038 /$ ncb2349

Pasero P, Vindigni A (2017) Nucleases Acting at Stalled Forks: How to Reboot the Replication Program with a Few Shortcuts. Annu Rev Genet 51:477-499. doi: 10.1146/annurev-genet120116-024745

Poli J, Tsaponina O, Crabbé L, et al (2012) dNTP pools determine fork progression and origin usage under replication stress. EMBO J 31:883-894. doi: 10.1038/emboj.2011.470

Remeseiro S, Cuadrado A, Carretero M, et al (2012) Cohesin-SA1 deficiency drives aneuploidy and tumourigenesis in mice due to impaired replication of telomeres. EMBO J 31:2076-89. doi: 10.1038/emboj.2012.11

Rhodes JDP, Haarhuis JHI, Grimm JB, et al (2017) Cohesin Can Remain Associated with Chromosomes during DNA Replication. Cell Rep 20:2749-2755. doi: 10.1016/j.celrep.2017.08.092

Ribeyre C, Zellweger R, Chauvin M, et al (2016) Nascent DNA Proteomics Reveals a Chromatin Remodeler Required for Topoisomerase I Loading at Replication Forks. Cell Rep 1-10. doi: 10.1016/j.celrep.2016.03.027

Robellet X, Vanoosthuyse V, Bernard P (2017) The loading of condensin in the context of chromatin. Curr Genet 63:577-589. doi: 10.1007/s00294-016-0669-0

Rudra S, Skibbens R V (2012) Sister chromatid cohesion establishment occurs in concert with lagging strand synthesis. Cell Cycle 11:2114-2121. doi: 10.4161/cc.20547

Samora CP, Saksouk J, Goswami P, et al (2016) Ctf4 Links DNA Replication with Sister Chromatid Cohesion Establishment by Recruiting the Chl1 Helicase to the Replisome. Mol Cell 1-14. doi: 10.1016/j.molcel.2016.05.036

Seeber A, Hegnauer AM, Hustedt N, et al (2016) RPA Mediates Recruitment of MRX to Forks and Double-Strand Breaks to Hold Sister Chromatids Together. Mol Cell 1-16. doi: 
10.1016/j.molcel.2016.10.032

Siddiqui K, On KF, Diffley JFX (2013) Regulating DNA replication in eukarya. Cold Spring Harb Perspect Biol 5:1-17. doi: 10.1101/cshperspect.a012922

Skibbens R V. (2016) Of Rings and Rods: Regulating Cohesin Entrapment of DNA to Generate Intraand Intermolecular Tethers. PLOS Genet 12:e1006337. doi: 10.1371/journal.pgen.1006337

Sogo JM, Lopes M, Foiani M (2002) Fork reversal and ssDNA accumulation at stalled replication forks owing to checkpoint defects. Science (80- ) 297:599-602. doi: 10.1126/science.1074023

Solomon DA, Kim J-SS, Waldman T (2014) Cohesin gene mutations in tumorigenesis: From discovery to clinical significance. BMB Rep 47:299-310. doi: 10.5483/BMBRep.2014.47.6.092

Stolz A, Hilt W, Buchberger A, Wolf DH (2011) Cdc48: A power machine in protein degradation. Trends Biochem Sci 36:515-523. doi: 10.1016/j.tibs.2011.06.001

Ström L, Lindroos HB, Shirahige K, Sjögren C (2004) Postreplicative recruitment of cohesin to doublestrand breaks is required for DNA repair. Mol Cell 16:1003-1015. doi: 10.1016/j.molcel.2004.11.026

Sutani T, Kawaguchi T, Kanno R, et al (2009) Budding yeast Wpl1(Rad61)-Pds5 complex counteracts sister chromatid cohesion-establishing reaction. Curr Biol 19:492-7. doi: 10.1016/j.cub.2009.01.062

Tercero JA, Diffley JF (2001) Regulation of DNA replication fork progression through damaged DNA by the Mec1/Rad53 checkpoint. Nature 412:553-557. doi: 10.1038/35087607

Thattikota Y, Tollis S, Palou R, et al (2018) Cdc48/VCP Promotes Chromosome Morphogenesis by Releasing Condensin from Self-Entrapment in Chromatin. Mol Cell 69:664-676.e5. doi: 10.1016/j.molcel.2018.01.030

Tittel-Elmer M, Alabert C, Pasero P, Cobb J a (2009) The MRX complex stabilizes the replisome independently of the $S$ phase checkpoint during replication stress. EMBO J 28:1142-56. doi: 10.1038/emboj.2009.60

Tittel-Elmer M, Lengronne A, Davidson MB, et al (2012) Cohesin Association to Replication Sites Depends on Rad50 and Promotes Fork Restart. Mol Cell 1-11. doi: 10.1016/j.molcel.2012.07.004

Tourrière H, Versini G, Cordón-Preciado V, et al (2005) Mrc1 and Tof1 promote replication fork progression and recovery independently of Rad53. Mol Cell 19:699-706. doi: 10.1016/j.molcel.2005.07.028

UhImann F (2016) SMC complexes: from DNA to chromosomes. Nat Rev Mol Cell Biol. doi: 10.1038/nrm.2016.30

UhImann F, Lottspeich F, Nasmyth K (1999) Sister-chromatid separation at anaphase onset is promoted by cleavage of the cohesin subunit Scc1. Nature 400:37-42. doi: 10.1038/21831

van den Boom J, Wolf M, Weimann L, et al (2016) VCP/p97 Extracts Sterically Trapped Ku70/80 Rings from DNA in Double-Strand Break Repair. Mol Cell 64:189-198. doi: 10.1016/j.molcel.2016.08.037

Verma R, Oania R, Fang R, et al (2011) Cdc48/p97 mediates UV-dependent turnover of RNA Pol II. Mol Cell 41:82-92. doi: 10.1016/j.molcel.2010.12.017

Villa F, Simon AC, Ortiz Bazan MA, et al (2016) Ctf4 Is a Hub in the Eukaryotic Replisome that Links 
Multiple CIP-Box Proteins to the CMG Helicase. Mol Cell 1-12. doi:

10.1016/j.molcel.2016.06.009

Wenzel ES, Singh ATK (2018) Cell-cycle checkpoints and aneuploidy on the path to cancer. In Vivo (Brooklyn) 32:1-5. doi: 10.21873/invivo.11197

Xiong B, Lu S, Gerton JL (2010) Hos1 is a lysine deacetylase for the Smc3 subunit of cohesin. Curr Biol 20:1660-1665. doi: 10.1016/j.cub.2010.08.019

Xu H, Boone C, Brown GW (2007) Genetic dissection of parallel sister-chromatid cohesion pathways. Genetics 176:1417-1429. doi: 10.1534/genetics.107.072876

Yazdi PT, Wang Y, Zhao S, et al (2002) SMC1 is a downstream effector in the ATM/NBS1 branch of the human S-phase checkpoint. Genes Dev 16:571-582. doi: 10.1101/gad.970702

Yeeles JTP, Janska A, Early A, Diffley JFX (2017) How the Eukaryotic Replisome Achieves Rapid and Efficient DNA Replication. Mol Cell 65:105-116. doi: 10.1016/j.molcel.2016.11.017

Zakari M, Yuen K, Gerton JL (2015) Etiology and pathogenesis of the cohesinopathies. Wiley Interdiscip Rev Dev Biol n/a-n/a. doi: 10.1002/wdev.190

Zou L, Elledge SJ (2003) Sensing DNA damage through ATRIP recognition of RPA-ssDNA complexes. Science 300:1542-8. doi: 10.1126/science.1083430

\section{Figure legends}

Figure 1. Structure of the SMC cohesin complex. Schematic representation of the structure of the core cohesin complex and its interacting factors. Names of budding yeast proteins and their human homologs (between parentheses) are provided. See text for further details.

Figure 2. A model for the control of cohesin function during the chromosome duplication cycle. Schematic model for the regulation of cohesin dynamic interaction with chromatin and its contribution to cohesin functions in DNA replication, stalled fork protection and chromosome segregation. Cohesin is loaded to chromatin prior to S-phase onset by the Scc2/Scc 4 complex. At this stage, cohesin interacts with DNA in a labile fashion due to the action of Wpl1 and Pds5, which promote the exit of embraced DNA molecules from the ring-shaped structure. During replication, a fraction of cohesin becomes stably associated to chromatin due to acetylation of two key lysines on Smc3, close to the DNA exit gate at its interface with Scc1. This modification is exerted by Eco1, which associates to replication forks, presumably once entrapment of the two sister chromatids has been achieved. Smc3 acetylation makes cohesin refractory to Wpl1/Pds5 function and establishes sister chromatid cohesion. Cohesion is maintained until its ultimate dissolution in anaphase through Smc3 deacetylation and Scc1 cleavage, mediated by Hos1 and separase, respectively. Cohesin mobilization and translocation from unreplicated chromatin to nascent duplexes is driven by Rsp $5^{\text {Bul2 }}$-dependent ubiquitylation and the action of the $\mathrm{Cdc} 48$ ubiquitin selective segregase. Cohesin translocation is required for the embracement and locking of nascent chromatids, which in turn 
promotes a stable configuration of replication machineries stalled in conditions of replication stress. Names of budding yeast factors are indicated. See text for further details. 
Figure 1

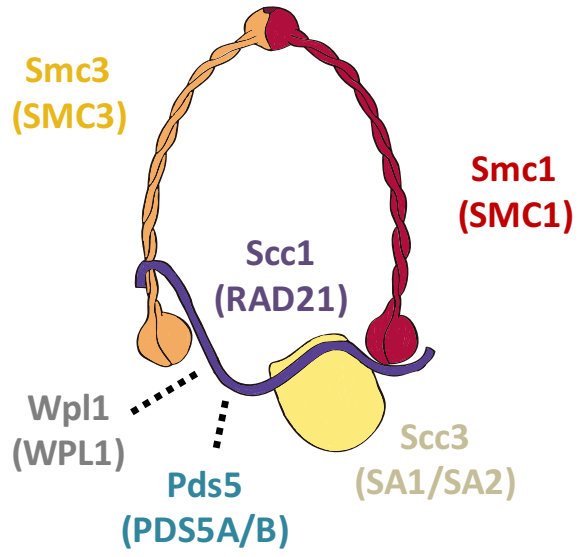

Figure 2

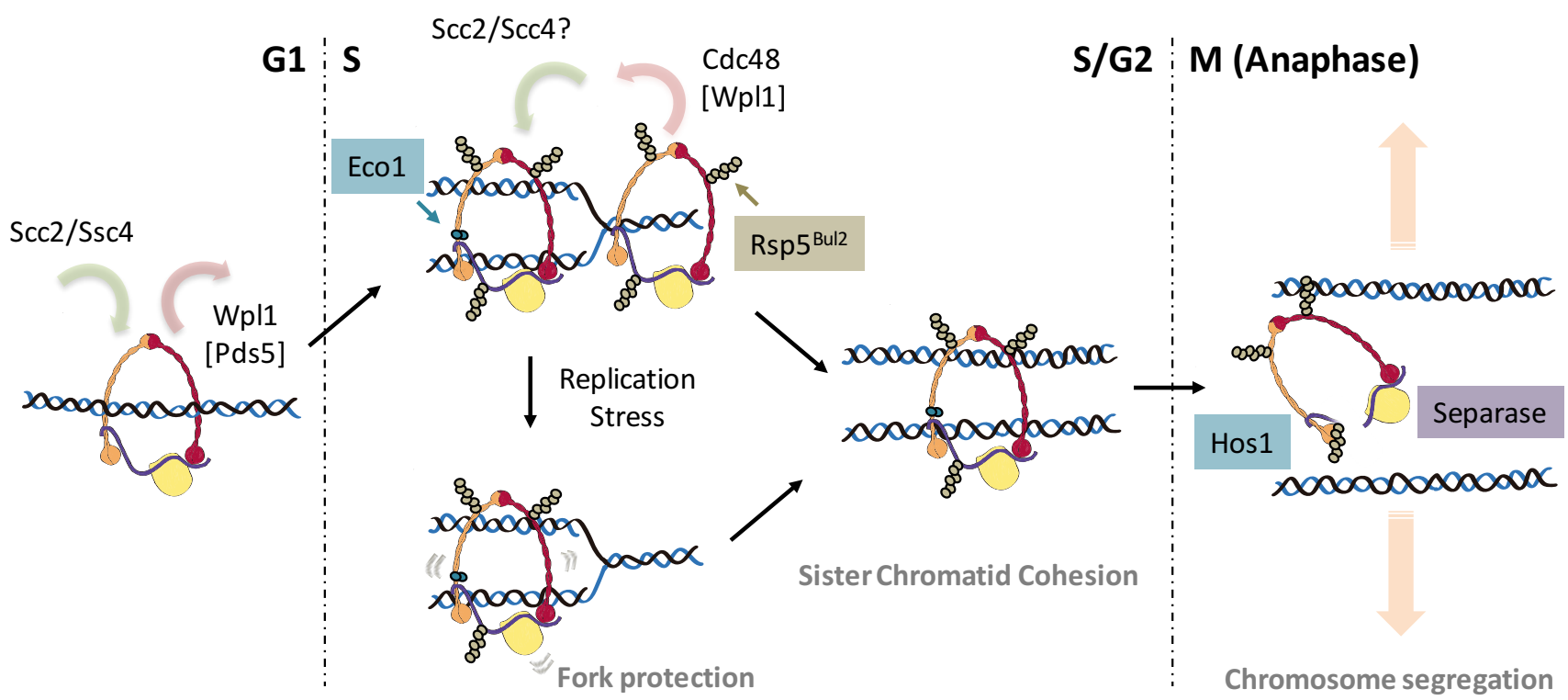

